\title{
ISSUES IN SYMBOL DESIGN FOR ELECTRONIC DISPLAYS OF NAVIGATION INFORMATION
}

\author{
Michelle Yeh and Divya Chandra \\ United States Department of Transportation Volpe National Transportation Systems Center, \\ Cambridge, Massachusetts
}

\begin{abstract}
An increasing number of electronic displays, ranging from small hand-held displays for general aviation to installed displays for air transport, are showing navigation information, such as symbols representing navigational aids. The wide range of display technology and the different functions these displays support makes it difficult to design symbols that are easily recognizable across platforms. The goals of this effort are to identify features of navigation symbology that are problematic when presented on electronic displays and to develop a method to design and evaluate symbology that takes into account the different media (e.g., paper vs. electronic) and platforms on which they will be displayed.

We address four questions to consider when evaluating the usability of a symbol: (1) Is the symbol easy to find? (2) Is the symbol distinctive from other symbols? (3) Is the on-screen symbol size appropriate? (4) Can all encoded attributes of the symbol be decoded quickly and accurately? Background findings and a proposed experiment to explore some of the higher-level issues related to the design of effective symbology are described.
\end{abstract}

\section{Introduction}

An increasing number of electronic displays are showing navigational information, i.e., information from aeronautical charts that assists the pilot in determining the aircraft's position. In a recently completed industry review, we found many different hardware platforms that support moving map or electronic chart applications. Some examples include an in-flight moving map display driven by a Flight Management System (FMS), an electronic chart on an Electronic Flight Bag (EFB), a surface moving map display on an installed unit, or a panel-mounted moving map display on a Global Positioning System (GPS) unit. The design of symbology for navigational displays is complex due to this wide range of display technology that can be used, but some level of commonality is important so that pilots do not misinterpret symbols as they transition between mediums (e.g., from paper charts to electronic map displays).

How a symbol looks on a display will vary depending on the physical qualities of the display. Fine symbols and special drawing conventions are used on paper charts to present a great deal of information in a relatively small space. However, paper-based symbols may be difficult to read when presented on electronic displays due to differences in display qualities.

There is currently no common standard across organizations and manufacturers regarding what symbols to show on electronic displays of navigation information. Standards for moving map displays are addressed by several organizations (e.g., [1], and [2]). Additionally, the Society of Automotive Engineers (SAE) has developed an Aerospace Recommended Practice (ARP) for electronic aeronautical symbols [3].

The goals of this effort are to identify the properties of usable symbology for electronic displays of navigation information, and to determine a rationale for choosing symbols, while taking into consideration the different media (paper vs. electronic) and platforms on which they will be displayed. In order to facilitate task performance, the symbol design must be able to directly convey the information represented, without inhibiting or interfering with the interpretation of other symbols [3]. We address issues to consider when determining what characteristics constitute usable symbology. Preliminary studies completed by the Volpe Center on this topic are presented briefly in this paper, and plans for an experiment are described.

\section{U.S. Government work not protected by U.S. Copyright}




\section{Characteristics of Usable Symbols}

The design and selection of symbols should consider the range of functions for which the display will be used. Symbols can be tested prior to use in order to determine their usability by measuring the performance impact against each of the following criteria:

- Is the symbol easy to find?

- Is the symbol distinctive from other symbols?

- Is the on-screen symbol size appropriate?

- Can all encoded attributes of the symbol be decoded quickly and accurately?

Usable symbols meet the criteria listed above. More detail on each of these criteria and methods for evaluating the performance impact are presented in the following sections.

\section{Is the Symbol Easy to Find?}

The salience of a symbol may be defined by how easy it is to find within a cluttered background. Saliency is influenced by many factors ranging from the context in which information is presented to the design of the symbol itself. Visual search for a symbol is heavily influenced by the number of items on the display (i.e., global density) as well as the number of items proximate to the target symbol (i.e., the local density). As more information is depicted on the display or as the number of items in close proximity to the target symbol increases, the saliency of the target symbol decreases. In general, the time it takes to find a target symbol increases linearly with increases in local and/or global density $[4,5]$. Since the local density surrounding a symbol and the global density on a chart vary widely from one chart to another, a symbol that may be easy to find in one context may be difficult to find in another. The time for finding a symbol could be nontrivial in time-critical situations, e.g., during final approach, when attention should be directed out the window rather than heads-down on the flight deck.

Search for a symbol may be facilitated if a symbol can be designed to be more salient than other items on the display. One way to make a symbol more salient is to manipulate attributes of the symbol, e.g., color or intensity. These differences are detected early and automatically in information processing, so that symbols presented in a unique color or intensity may appear to "pop out" of the display. Hence, such techniques mitigate the time for visual search as the number of items on the display increases [6]. However, color should not be used excessively in an effort to make symbols distinct. Color perception varies across individuals, but generally, users cannot accurately classify more than seven different levels of color or intensity at once. These limits in classification will become greater in conditions of glare or low illuminations [7].

Although color and intensity are currently used on paper charts, they are used only to increase the contrast between display elements rather than to code important differences in navigation symbols. For example, on approach charts or low altitude enroute charts, color is typically used for the background or to code additional information such as terrain. Similarly, intensity differences are used simply to distinguish between features, e.g., on an airport diagram, runways are presented at a higher intensity than other movement areas.

In the future, color and intensity differences could be used to enhance the salience of certain symbols and to reduce the salience of others. A color philosophy for the design of electronic charts has not yet been established. In defining a coding scheme, it will be necessary to determine how display elements should be grouped and to evaluate what colors should be used for different symbols. Symbols that need to be integrated should be presented in a similar color or intensity but in a different color or intensity from symbols that are processed independently $[4,5]$. For integrated systems, it may be important to consider color conflicts that may result, e.g., yellow is often used to represent cities on charts for visual operations, but is used for caution indicators on other displays.

A good symbol is not only salient, but also distinctive, so that it is easy to recognize. Note that the task of recognition only requires the pilot to match the symbol on the chart with what is in his/her stored memory. Characteristics of a symbol that make it distinctive are discussed in the next section. 


\section{Is the Symbol Distinctive?}

A symbol on a moving map display is distinctive if it is easy to discriminate from other symbols, even if it differs from other symbols by only one feature. Symbols are designed to have a basic shape or characteristic that can be recognized by users.

The distinctiveness of a symbol may be measured by assessing the degree to which it can be identified outside the context of a chart. If the symbol is identifiable within the chart, then contextual clues (e.g., the location of the symbol on a chart) could be used to determine what the symbol represents, if the meaning is not obvious. By removing all contextual clues, it is possible to discern the meaning conveyed by the symbol itself.

It is also important to examine the distinctiveness of a symbol across symbol sets, e.g., compare symbols used by different system designers. Two factors should be considered: (1) the consistency of a symbol design, i.e., the basic symbol shape should be recognizable regardless of the chart provider, and (2) the confusability of a symbol with other symbols. Each of these issues is considered below.

\section{Consistency}

Consistency in symbol design across chart providers will facilitate interpretation of the symbols. However, the design of electronic display symbols has historically resulted in differences from one manufacturer to another. [3] presents a comparison of symbol sets and provides recommendations for electronic aeronautical symbols. However, informal discussions with manufacturers suggest that the recommended SAE symbols are not in widespread use. One reason is some of the symbols require a level of detail that is not possible on low resolution displays. Another is that some of the recommended symbols are similar to copyrighted symbols; consequently, some manufacturers are wary about using them.

A recent survey of technology conducted by the Volpe Center compared symbols from eight different manufacturers with those listed in [3]. The comparison highlighted (1) the use of non-standard symbols, particularly for lower end displays that may have lower resolution capabilities, and (2) varying levels of detail conveyed by the symbols depending on the manufacturer. Thus, the potential for confusing and misleading symbology exists.

An example, comparing the representation of the Distance Measuring Equipment (DME) symbol, is shown in Table 1 below. The DME symbols in the table are in use by the United States (US) government's Federal Aviation Administration (FAA) National Aeronautical Charting Office (NACO), ICAO, and recommended by SAE. The US and ICAO symbols are almost identical, but both differ significantly from the SAE recommended symbol.

Table 1. Variations in the DME Symbol

\begin{tabular}{|l|l|l|l|}
\hline & $\begin{array}{l}\text { US } \\
\text { Symbol }\end{array}$ & $\begin{array}{l}\text { ICAO } \\
\text { Symbol }\end{array}$ & $\begin{array}{l}\text { SAE } \\
\text { ARP 5289 }\end{array}$ \\
\hline DME & $\square$ & $\square$ & $\{\because$ \\
\hline
\end{tabular}

A consistent appearance in the presentation of aeronautical chart symbology from one system to another facilitates pilots' transition and crosschecking between systems. Consistency in the presentation of important information is maintained on paper charts because the pilot carries them from one aircraft to another. The issue of consistency in data presentation and compatibility across electronic systems will be more important as electronic charts replace existing paper charts in the future. When designing new symbols, it is important to consider familiarity with the charts, i.e., conventions for paper charts, and the potential training impact of introducing new symbols. For example, pilots using an FMS display may confirm information by looking to a paper chart.

Symbol recognition is determined by its key defining features, i.e., features unique to that symbol. For example, if the key feature for the DME symbol is its overall shape, will pilots who are familiar with ICAO symbols recognize the SAE DME symbol? Pilot experience and training may affect which symbols are easiest to recognize and use since familiarity with symbols will influence how new symbols are interpreted. Consequently, the usability of a symbol set may differ depending on whether the target population is general aviation or air transport. 


\section{Confusability}

Inconsistency in how a symbol is represented across chart providers can produce cases where a symbol from one provider may be confused with a symbol from a different provider. This confusion may result when symbols from two chart providers are visually similar but used in different ways. For example, in 1999, the FAA identified the potential for confusion due to the similarity between the US representation for a fly-by waypoint and the (now out of date) ICAO representation for a fly-over waypoint. The symbols that were in use then are shown in Table 2.

Table 2. US and ICAO Fly-By and Fly-Over Waypoint Symbols

\begin{tabular}{|l|l|l|}
\hline & US Symbols & $\begin{array}{l}\text { Previous } \\
\text { ICAO } \\
\text { Symbols }\end{array}$ \\
\hline $\begin{array}{l}\text { Fly-By } \\
\text { Waypoint }\end{array}$ & 0 \\
\hline $\begin{array}{l}\text { Fly-Over } \\
\text { Waypoint }\end{array}$ & 0 & \\
\hline
\end{tabular}

The fly-by waypoint symbol used by NACO and the fly-over waypoint symbol previously recommended by ICAO were both four-pointed stars with filled points. The operational meanings for a fly-by waypoint versus a fly-over waypoint are significantly different, however. A fly-by waypoint allows the pilot to anticipate a turn to avoid overshooting the next flight segment. A fly-over waypoint requires crossing over the waypoint prior to initiating a turn. Fly-over waypoints are usually designated because of an obstacle clearance requirement. If these symbols are misinterpreted by a pilot, the resulting flight path deviation could have safety implications.

The Volpe Center was asked to provide input on this conflict. An exploratory paper-based study to evaluate the saliency of the fly-over and fly-by waypoint symbols in a cluttered chart context was conducted. Saliency was used as a measure because it is important that the selected symbols are easy to find. In particular, the fly-over symbol should be salient since it may be used when there is an obstruction to safe flight.
Three symbol sets were evaluated: the US and previous ICAO symbols, shown in Table 2 and an alternative proposed by the International Air Transport Association (IATA), shown in Table 3.

Table 3. IATA Fly-Over and Fly-By Waypoint Symbols

\begin{tabular}{|l|c|}
\hline & IATA Alternative \\
\hline $\begin{array}{l}\text { Fly-Over } \\
\text { Waypoint }\end{array}$ & 0 \\
\hline $\begin{array}{l}\text { Fly-By } \\
\text { Waypoint }\end{array}$ & O \\
\hline
\end{tabular}

Pilots were asked to find fly-over and fly-by waypoint symbols in mock paper charts, which were modifications of real NACO charts. A practical constraint of the NACO format was that the symbol size varied, because NACO uses two symbol sizes. The standard fly-over or fly-by symbol is approximately $5 \mathrm{~mm}$ in diameter in the plan view, but, if a waypoint symbol is drawn at the runway, its size is reduced to approximately $3 \mathrm{~mm}$ so that the runway remains visible. The difference in symbol size applied only to the presentation of the fly-over waypoints; no small fly-by waypoints were shown in the mock charts.

The saliency of a symbol was assessed by comparing the accuracy with which it was detected. Accuracy was calculated as a percentage of the number of symbols detected relative to the total number of symbols in the chart. Note that the study was performed against external time constraints, so data from only 11 air transport pilots (some of whom had experience with the NACO format through prior military flight experience) was collected. Results showed that (1) the ICAO fly-by waypoint symbol was detected more accurately than the NACO and IATA fly-by symbols in the mock charts, and (2) the ICAO fly-over waypoint was detected less accurately than the NACO and IATA fly-over waypoints. The poorer performance in detecting the ICAO fly-over waypoint was primarily attributable to problems detecting the symbol when it was small. Because the small flyover waypoint is shown near the runways, adding the circle may have increased the saliency of the fly-over waypoint by providing a feature (the arc 
shape) to distinguish the symbol from the runways and arrows, composed of straight-line segments.

In 2000 , ICAO addressed the inconsistent implementation of waypoint symbology by adding a circle to the recommended fly-over waypoint symbol (see [1]). The waypoint symbols recommended by ICAO now resemble the IATA alternative, shown in Table 3. The addition of a circle is especially important because ICAO does not specify a minimum symbol size for the symbol; the circle could be especially helpful when the symbol is small.

Not surprisingly, large symbols are easier to detect and discriminate than smaller symbols, but when display space is limited, a symbol cannot be too large. When designing and evaluating symbols, it will be important to consider whether a minimum size should be specified, and how this minimum size may be influenced by characteristics of the display technology, as described in the following section.

\section{Is the On-Screen Symbol Size Appropriate?}

The minimum size at which a symbol is presented must preserve the key features that define it. Detail in a symbol is more difficult to distinguish on a small symbol than on a large symbol, particularly if resolution is limited. For example, the filled region in the previous ICAO fly-over waypoint symbol (shown below in Table 4), which distinguishes the fly-over waypoint symbol from the fly-by symbol, becomes less salient on an electronic display as the symbol size is reduced. (Note that differences in the symbol may still be noticeable since the symbols are drawn at $600 \mathrm{dpi}$ on paper here).

Table 4. Effect of Symbol Size on Detail.

\begin{tabular}{|l|l|l|}
\hline & $5 \mathrm{~mm}$ & $3 \mathrm{~mm}$ \\
\hline $\begin{array}{l}\text { Fly-Over } \\
\text { Waypoint }\end{array}$ & $\diamond$ & $\diamond$ \\
\hline $\begin{array}{l}\text { Fly-By } \\
\text { Waypoint }\end{array}$ & $\mathrm{A}$ & $\diamond$ \\
\hline
\end{tabular}

The appearance of a symbol - - and the level of detail that can be distinguished - will depend on the qualities of the display technology on which it is presented. Electronic displays that show navigation information vary widely, as noted earlier. In general aviation, the biggest difference between low-end and high-end displays is resolution. Resolution is the number of pixels available on the display; as resolution increases, the image appears to be more defined. At low resolutions, symbols may be more easily confused because discriminating features may not be depicted clearly. Hence, the minimum physical size that a symbol can be drawn will vary depending on the display resolution. For example, an $800 \times 600$ pixel resolution display is suitable for text but may not be as suitable for small fonts and intricate graphics. As resolution increases, symbols may be identifiable at smaller sizes.

In the air transport domain, we found that although display resolution was still important in determining a symbol's appearance, it was not a limiting factor; in fact, the resolution of what was considered a low-end display for air transport aircraft was higher than the resolution for some high-end displays used in general aviation operations. Rather, the important issue for air transport displays is optimizing contrast, e.g., by increasing luminance or stroke width. One manufacturer described creating symbols on air transport displays as drawing with a thick crayon. Consequently, a symbol with a high level of detail will be difficult to draw.

In addition, it is important to note that, in the air transport domain especially, software upgrades and new functions and applications may be implemented on a display without replacing the existing installed hardware or processors. For example, electronic moving map functions with new symbols could be presented on FMS displays. As a result, symbol design may be constrained by the capabilities of current installed display hardware and processors.

Symbol size is closely tied to the issue of display size. Larger displays allow symbols to be drawn using a greater number of pixels than small displays, e.g., a 3-inch square display. Electronic displays that present navigation information may be very small. These displays may have no more than an $80 \times 240$ resolution. Consequently, some small symbols may be difficult to see at the normal viewing distance.

Limitations in display capabilities can be addressed by determining the minimum size a 
symbol can be presented across a range of display resolutions. The evaluation of a symbol must consider how small the symbol can be before fine details cannot be distinguished and its usability is impacted. If symbols from paper charts are being adapted for use on electronic displays, it will be necessary to determine how much, if any, detail will be lost in the conversion of the symbols from paper to electronic format. For example, symbols used on FMS displays typically have less detail than symbols used on paper (see [3] for examples). For each symbol, it will be necessary to determine the minimum size at which it can be presented so that it is easily discriminable and identifiable.

Such a study was conducted by the Volpe Center in support of the efforts of the SAE G-10 Aeronautical Charting Subcommittee to develop a recommended symbol set prior to the 1997 publication of [3]. Participants were shown proposed symbols on a computer screen and asked to adjust the symbol to the smallest size at which it was still recognizable. The symbols were shown across a range of display resolutions and environmental factors (i.e., with and without vibration). The results were used to identify key features for each symbol, and to provide recommendations for the minimum size at which each symbol should be presented so that the key features would be easily discriminable.

It may be possible to vary symbol detail depending on the symbol size. One manufacturer, for example, creates symbols in two sizes - a smaller, less detailed image that is shown at high map ranges and a larger, more detailed image that is shown when the user zooms in on the display. This allows the symbol size to appear appropriate in context. If the symbol size were fixed, then at high map ranges (i.e., when the user is zoomed out), the symbol could appear relatively large and could overlap with other symbols in close proximity resulting in clutter.

The distinctiveness of the symbol detail across all sizes at which the symbol will be presented becomes particularly important as the symbol design increases in complexity. While high levels of detail may convey more information about a target symbol, the additional complexity may inhibit the usability of the symbol. In the next section, the effects of complexity on the usability of the symbol and the extent to which users can interpret symbol meaning as symbols become more complex are addressed.

\section{Can All Encoded Attributes of the Symbol Be Decoded?}

Symbols may be integrated so that one symbol will have multiple attributes that encode information about the object. For example, symbols for navigation aids and reporting points may be combined by using the symbol shape to represent the type of navigation aid and the symbol fill to represent whether that navigation aid is a compulsory reporting point or not. Recently, the US submitted a proposal to ICAO to establish a charting hierarchy so that there is a consistent method for distinguishing between ground-based points and GPS/Area Navigation (RNAV) points for navigation purposes. The proposal also recommends a consistent way to modify symbols to distinguish between compulsory versus on-request reporting and between fly-over versus fly-by requirements [8]. An example is shown in Table 5.

Table 5. Symbols Defined by Combining Shape and Fill

\begin{tabular}{|l|c|c|}
\hline $\begin{array}{l}\text { Symbol shape: } \\
\text { Navigation } \\
\text { equipment }\end{array}$ & $\begin{array}{l}\text { Unfilled }= \\
\text { On-request }\end{array}$ & $\begin{array}{l}\text { Fill } \\
\text { Filled }= \\
\text { Compulsory }\end{array}$ \\
\hline $\begin{array}{l}\text { Triangle }= \\
\text { Ground-based }\end{array}$ & $\Delta$ & $\Delta$ \\
\hline $\begin{array}{l}\text { Waypoint }= \\
\text { RNAV }\end{array}$ & & \\
\hline
\end{tabular}

As Table 5 shows, the symbol shape designates the navigation-equipment requirement. A triangle symbol identifies the location of fixes and intersections, defined by ground-based navigation aids, and the four-pointed waypoint star identifies the location of RNAV waypoints, defined by coordinates as points in space. The shape attribute is combined with fill to indicate whether the point is an on-request or compulsory reporting point. An unfilled triangle represents on-request reporting at a ground-based point, and a filled triangle represents compulsory reporting at a ground-based point. A third attribute, shown earlier in Table 2, is the presence or absence of a circle that surrounds the 
symbol. The circle differentiates between a fly-over requirement (circle) and a fly-by requirement (no circle).

When expanding the definitions of current symbols and/or integrating symbols, the design of the symbol needs to be evaluated to determine whether the meaning conveyed by the combined attributes is clear and intuitive. The results of an exploratory study conducted at the Volpe Center caution that symbol features rules may not be a strong cue for pilots in identifying unfamiliar symbols. In the study, attributes for reporting point and waypoint symbols (e.g., shape and fill) were combined to produce a set of test symbols. The 11 air transport pilots who participated in the earlier study, examining fly-by and fly-over waypoints, were also presented with a legend defining the basic symbols and asked to rate their confidence in classifying the test symbols as (1) fly-over or fly-by waypoints and (2) on-request or compulsory reporting points. The test symbols could be real, i.e., chart symbols that pilots were familiar with that did not actually appear in the legend, or fake, i.e., symbols that were not based on any of the legend symbols nor were they associated with any other type of chart symbol. Pilots' confidence in their ability to classify the fake symbols provided an assessment of the ability to generalize the symbolfeature rules. The results showed that while pilots were able to determine the meaning of symbols based on information in the legends, they did not have confidence in their abilities to generalize the symbol-feature rules broadly.

The extent to which symbol-feature rules are applied consistently will affect the learnability of the symbol. If there are many exceptions, the symbol-feature rule may have limited generalizability and will not be learned [7]. Additionally, it will also be important to consider whether the application of the symbol-feature rule is consistent with previous training. That is, if the definition of a symbol is being expanded, then the new definition of the symbol should not be inconsistent with the current use of the symbol.

\section{Next Steps}

This paper sets forth a set of issues for research. Research examining how a symbol should be designed is relatively new in the aviation domain where symbols are usually created by manufacturers without any formal human factors testing. We are pursuing independent studies to understand the higher-level issues related to the design of effective symbology. This research will support ongoing independent efforts by SAE and the FAA to construct a symbol set.

Our first goal is to determine what key features are necessary for symbol recognition. A review of recommended symbols listed in [3] show inconsistencies across organizations and manufacturers. Key features defined in [3] are not always salient in symbols used by manufacturers. Additionally, the relationship between symbols used on paper charts and those used on electronic displays will be addressed. Should display limitations be considered in the design of paper symbols to ensure symbol consistency in the transition from paper to electronic displays?

A second goal is to investigate the usability of symbols if their meaning is redefined and as their design becomes more complex. Proposed rules for encoding symbol attributes will be evaluated to determine how intuitive the rules are in helping pilots learn the symbols' meanings.

\section{Proposed Experiment}

The experiment will consist of four different tasks. For each task, participants will be shown a series of symbols without context and asked to answer one of the following questions:

- What is the symbol?

- Does the symbol depict a fly-by or flyover requirement?

- Does the symbol depict a compulsory or non-compulsory reporting point?

- Is it a ground-based or RNAV point?

Our dependent variables will be the response time needed to answer each question, the response accuracy, and a rating of the participants' confidence in the response. Considerations for the experiment design are discussed below.

\section{Participants}

Pilots' background and training will influence their familiarity and interpretation of symbols. Participants may be from one of three groups: air 
transport pilots who fly in the continental US, air transport pilots who fly transatlantic flights and therefore have more experience with compulsory reporting, and GA pilots who fly in the continental US.

\section{Symbols}

Three symbols types (NAVAID, triangle, and waypoint), shown in Table 6 below will serve as base symbols.

Table 6. Base Symbols

$\begin{array}{cl}\Delta & \text { Fix/Intersection } \\ 0 & \text { VOR } \\ \square & \text { VOR/DME } \\ \square & \text { TACAN } \\ 0 & \text { NDB } \\ 0 & \text { VORTAC } \\ \beta & \text { Waypoint }\end{array}$

Each symbol may be modified in two ways: fill (as shown in Table 5) to designate compulsory vs. on-request reporting, and the presence or absence of a circle to designate fly-over vs. fly-by. Distractor symbols will also be shown to determine whether or not participants can apply the rules to other symbols and to determine whether new symbols created by the charting hierarchy will be confusable with symbols currently used by other chart providers.

\section{Symbol Legend}

The amount of instruction that participants are given about how the symbol rules are applied will influence their ability to infer the rules. The level of detail in a legend may vary from minimal to detailed. A minimal legend would provide one or two examples of the rule without explicit instruction, while a detailed legend would provide explicit, detailed description of the rules and many examples.

\section{Applications}

The results of this experiment are expected to facilitate the transition from paper charts to electronic displays of navigational information. The results will provide input to the FAA on the usability of the proposed charting hierarchy. In addition, this research will support the efforts of the SAE G-10, Aerospace Behavioral Engineering Technology Committee, Electronic Charting Subcommittee in its upcoming effort to update [3]. While the scope of this work addresses navigation symbology, the techniques used here are applicable for addressing the usability of other types of symbology.

\section{Acknowledgements}

This research is being conducted by the Volpe Center's Operator Performance and Safety Analysis Division and was funded by the Human Factors Research and Engineering Division of the FAA. We would like to thank Tom McCloy, our FAA program sponsor, for his support and guidance. Input from the FAA Office of Aircraft Certification and the FAA National Aeronautical Charting Office was also critical.

\section{References}

[1] ICAO Annex 4. Aeronautical Charts, Annex 4 to the Convention on International Civil Aviation, 10th edition, July 2001.

[2] RTCA. (2003). Minimum Operational Performance Standards for the Depiction of Novigational Information on Electronic Maps. DO257A. RTCA: Washington, D.C.

[3] Society of Automotive Engineers (SAE). (1997). Electronic Aeronautical Symbols, ARP 5289. Society of Automotive Engineers: Warrendale, PA.

[4] Christ, R.E. (1975). Review and analysis of color coding research for visual displays. Human Factors. 17(6). p. 542-570.

[5] Teichner, W.H. and J.B. Mocharnuk. (1979). Visual search for complex targets. Human Factors. 21(3), 259-275.

[6] Kahneman, D. and A. Treisman. (1984). Changing views of attention and automaticity. In R. Parasuraman \& D.R. Davies (Eds.), Varieties of Attention. Orlando, FL: Academic Press.

[7] Wickens, C.D. and J.G. Hollands. (2000). Engineering Psychology and Human Performance, 3rd edition. Upper Saddle River, NJ: Prentice Hall. 
[8] ICAO Aeronautical Information and Charts Study Group Meeting. Working paper. Concept for RNAV/Ground-Based Charting Symbol

Consistency and Hierarchy. 3-5 June, 2004.

Montreal, Canada.

\section{Disclaimer}

The views expressed herein are those of the authors and do not necessarily reflect the views of the Volpe National Transportation Systems Center, the Research and Special Programs Administration, or the United States Department of Transportation. 